\title{
What variability tells us about motor expertise: measurements and perspectives from a complex system approach
}

\author{
John Komar ${ }^{1,2}$, Ludovic Seifert ${ }^{2}$ and Régis Thouvarecq ${ }^{2}$ \\ 1 Performance.LAB, Prozone Sport, Leeds, United Kingdom \\ 2 CETAPS, Département des Sciences du Sports, Normandie Université, Boulevard Siegfried, 76821 Mont-Saint-Aignan, Rouen, \\ France
}

Received 4 September 2014 - Accepted 17 April 2015

\begin{abstract}
In this review, we revisit the concept of movement variability in motor expertise from the perspective of complex systems theory; we do so by defining performers and their relationships with the environment. The complex systems perspective places the focus on the functional role of movement variability as an emergent response to interacting constraints as performers pursue taskgoals. We therefore show how the shift in theoretical focus towards the functional role of variability has entailed a reconsideration of the way this key property of motor expertise should be assessed. We then present the main theoretical issues surrounding movement variability and expertise through examples of empirical applications of the relevant tools and concepts in the sport and movement sciences.
\end{abstract}

Key words: Expert performance, adaptability, degeneracy, cluster analysis, recurrence quantification analysis

Résumé. Ce que la variabilité motrice nous apprend sur l'expertise : mesures et perspectives du point de vue des théories de la complexité.

À travers cette revue, nous revisitons le concept de variabilité motrice et sa relation à la notion d'expertise à partir d'un point de vue emprunté aux théories de la complexité. Pour cela, nous nous intéressons dans un premier temps à un niveau général qui décrit la relation qu'entretient un sujet avec son environnement. Au sein de cette relation sujet-environnement, une perspective issue des théories de la complexité porte alors une attention toute particulière au rôle fonctionnel de la variabilité motrice comme propriété émergente de l'interaction de contraintes pesant sur le sujet. À partir de ce postulat, nous proposons ensuite de reconsidérer la manière dont la variabilité motrice est évaluée à partir de l'utilisation de statistiques non-linéaires ou non-supervisés, ceci afin de répondre à cette acception de la variabilité comme élément clé de l'expertise motrice. Enfin, nous présentons quelques exemples de questionnements théoriques soulevés par notre positionnement à travers un approfondissement d'études empiriques réalisées dans le domaine du sport et des sciences du mouvement humain.

Mots clés : Performance experte, adaptabilité, dégénérescence, statistiques non-supervisés, analyses de récurrence

In the field of motor control and performance research, the question of motor variability inevitably arises. However, although researchers acknowledge its existence, the status of variability as a motor skill has been subject to debate (e.g. Newell \& Corcos, 1993). Indeed, according to the ontological and epistemological framework (Bachelard, 1971; Zwirn, 2006) within which motor control is usually studied, variability has been envisaged in a quite radically different way. The first framework for studying motor control was borrowed from the field of cybernetics (information processing) (Fitts, 1964; Shannon \& Weaver, 1949), and it has dominated for at least 50 years. This cognitivist perspective was based on the analogy drawn between the computer (a physical machine computing information) and the brain (a biological machine computing information) (Varela, 1989). Motor production (i.e. all the organized muscular contractions directed towards accomplishing a goal) was thus seen as the consequence of generalized motor programs (GMP), the source of which was assumed to be at a 
higher level: the central nervous system. In this traditional approach, the motor program is made up of an invariant part, the plan (common to all movements having an identity of shape), and the parameters that allow the plan to be adapted to a given context (Schmidt, 1975), thereby ensuring flexibility when the environment fluctuates. From this perspective, good performance is reproducible performance when the context is constant, and variability is detrimental or indicates a weakness in the performance (Davids, Glazier, Araújo, \& Bartlett, 2003; Newell, 1991). This approach considers variability in a given movement pattern to be the result of error (Stergiou \& Decker, 2011), reflecting an inability to predict the parameters needed to employ the underlying motor program (Schmidt, 2003). By extension, motor learning is the gradual reduction and ultimately the elimination of variability in order to optimize the accuracy and efficiency of movement patterns. Yet in practice, movement variability is observed even in highly skilled performers, originating from both internal physiological processes (e.g. genes, ion channels, neuromotor transmission, control level) and the environment (e.g. opponents, partners in team sports, weather conditions in outdoor sports) (Muller \& Sternad, 2004).

Despite the undeniable scientific power of this cognitivist approach [Shea and Wulf (2005) reported 782 citations of Schmidt $(1975)^{1}$ between 1975 and the end of 2004)], it has come under challenge since the 80s as new approaches have emerged, many of which have been brought under the general umbrella of complex systems science (Davids et al., 2014). According to von Bertalanffy (1969), a complex system is a set of units in mutual interrelationship. A key assumption is that both the high number of elements and the many relationships among them give rise to the complexity. De Rosnay (1975) then added that a complex system is a set of elements in dynamic interaction, organized around a goal. Although no single definition of a complex system has ever been unanimously agreed on (Prigogine, 1994), living systems are undeniably complex (from com-plexus, the property of something that is woven together). Unsurprisingly then, complex system characteristics like selforganization, emergence, sensitivity to initial conditions and nonlinearity have been highlighted in the relevant movement science literature (Haken, Kelso, \& Bunz, 1985; Kelso, 1984; Kugler, Kelso, \& Turvey, 1980). The complex systems framework therefore encapsulates the concepts and tools from both Coordination Dynamics (Kelso, 1995) and Ecological Psychology (Gibson, 1979) in order to put the focus on the key parameters that describe system organization (i.e. order parameters) and the variables that act as information to constrain the way systems adapt to key environmental objects and tasks (i.e. control parameters). In addition to focusing on the emergent

1 The schema theory (Schmidt, 1975) is probably the best known cognitivist model in sport science research, although other models exist (e.g. Wolpert, 1997). property of behaviour, the complex systems approach supports the work of Gibson (1979), who posited that individuals relate to their environment by direct informational coupling without the mediation of symbols (Shaw, 2003). From the complex systems perspective, movement variability is not perceived as detrimental or as the reflection of inconsistency in a motor skill, but rather as a key signature of adaptability (Seifert, et al., 2013). As Biryukova and Bril (2002) noted, dexterity does not refer to the movement itself, but rather to the ability to adapt to external constraints (p. 65), a comment that underlined the growing interest in investigating variability in neurobiological systems (Newell \& Corcos, 1993).

Ericsson, Krampe and Tesch-Römer (1993), who investigated motor expertise from a cognitivist or traditional perspective, operationally described an expert as an individual that has accrued at least ten years or $10,000 \mathrm{~h}$ of deliberate, high-level practice. They proposed that learners exposed to such a period of intense, repetitive practice would thus develop the capacity to reproduce the same behaviour and with an automatic control of movement. The main goal of deliberate practice is therefore to reduce any deviation from an internalized expert model in order to produce optimal methods for task performance (see et al. 2013). From a complex systems perspective, however, interest is not restricted to the question: "what are the stable states exhibited by experts?" after these intense repetitions, but expands to include such questions as "what are the sources of variability?', 'is variability associated with expertise?", and "how is variability controlled while learning a new skill?" (Stergiou \& Decker, 2011, p. 870). For example, Kelso, DelColle, Schöner (1990) highlighted that the variability in coordination observed at the approach of transition zones provides an opportunity to explore the various patterns that may be adopted. This idea of behavioural variability having a functional role has been proposed in studies of posture (Riley, Balasubramaniam, \& Turvey, 1999), sport expertise (Seifert, et al., 2013) and motor learning (Nourrit, Delignières, Caillou, Deschamps, \& Lauriot, 2003).

The advantage of this theoretical perspective is that great attention is given to behavioural variability, using less traditional and even original statistical tools, in order to highlight key properties of motor variability and expertise. The next parts of this review will therefore provide, i) a brief review of the tools used to analyse movement variability, and ii) the theoretical assumptions underlying the research on movement variability and motor expertise by presenting empirical applications in human movement studies.

\section{Perspectives in measuring motor variability}

Variability is intrinsic to all biological systems and can be observed quite easily. Independently of the nature of 

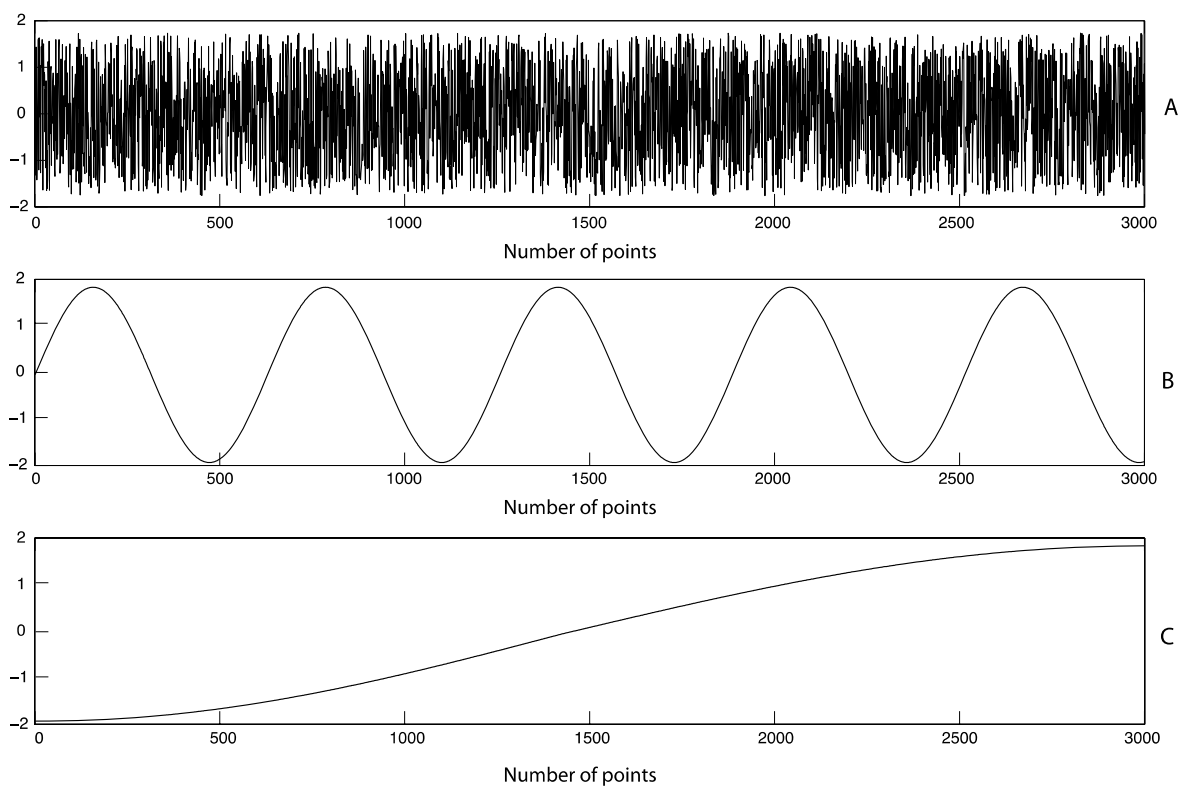

Fig. 1. Examples of a sinusoidal time series with a mean $=0$ and a standard deviation $=1$ (B) (scale is arbitrary): the same points re-ordered in random order (A) and in ascending order (C).

the measure and the level of investigation (e.g. neurobiological, behavioural or social; Mason, 2010), variability can be defined as 'the variance of movements generated by an individual under the same task conditions' (Newell \& Slifkin, 1998, p. 143): if someone tries to perform the same movement twice, the two movements will never be completely identical. Movement variability can further be defined as intra-trial (e.g. variability in the position of the centre of pressure over time when standing, Riley \& Turvey, 2002), inter-trial (e.g. variability in knee and elbow coordination before and after a given practice program) (Komar, Hérault, \& Seifert, 2013), or inter-subject (e.g. variability in the coordination patterns of football players with different levels of expertise; Chow, Davids, Button, \& Koh, 2008). Two main types of analysis can thus be used to define movement variability: i) timecontinuous data analysis to study intra-trial variability and ii) discrete data analysis to study inter-trial or intersubject variability. In the first case, the aim of the analysis is to define or characterize the nature of movement variability within a trial, whereas the second case refers to a comparison between trials from different practice conditions or between different individuals.

\subsection{Time-continuous data analysis}

In the intra-trial context, movement variability has traditionally been measured in terms of the statistical variance or standard deviation in the time evolution of the variable (e.g. movement outcome, kinetics, kinematics). For example, the standard deviation is useful to characterize the amount of variability present in the perceptual-motor system, as it quantifies the mean amount of deviation (or dispersion) around an averaged value. The main problem with this indicator is that in some cases like the study of a fixed point in a dynamical system, it does reflect an estimation of the stability of this system, but in other cases (e.g. chaotic attractors, limit cycle attractors) different time series can exhibit both the same mean and the same standard deviation even if they do not share the same structure in time. As an example, Figures 1A, $1 \mathrm{~B}$ and $1 \mathrm{C}$ present the same mean value $(M=0)$ and the same standard deviation $(\mathrm{sd}=1)$, but obviously do not represent the same characteristics. In fact, Figures $1 \mathrm{~A}$ and 1C contain the same points of the sinusoid of Figure 1B, but their initial time stamp is simply reorganized in random order (1A) or ascending order (1C). Figure 1A represents a stationary random process (i.e. despite knowing the previous point, it seems difficult if not impossible to determine the next point), whereas Figure 1B represents a stationary deterministic process (i.e. knowing the previous point, it seems easy to determine the next point) and Figure $1 \mathrm{C}$ represents a non-stationary process (i.e. the structure of the process evolves with time).

Obviously, for an in-depth investigation of variability, "the order of data points matters" (Kuznetsov, Bonnette, \& Riley, 2014, p. 83). Therefore, a complex systems approach in movement science implies more than the simple quantification of variability; instead, it emphasizes the importance of investigating the embedded structure and nature of this variability. To assess the fundamental properties of time series, the assumption is made that they are chaotic processes, which means that they reflect both a strong determinism and a (quasi-)impossibility for prediction (Fonseca, Diniz, \& Araújo, 2014). More precisely, the high sensibility to initial conditions (a.k.a. the 
"butterfly effect") makes the behaviour hardly predictable because a small change in the conditions of practice (e.g. a $0.5 \mathrm{~m}$ change in the position of only one teammates in a football game) can lead to a completely different output (e.g. the player in possession of the ball shooting and scoring instead of passing). The system is determinist as the same situation always leads to the same outcome, but the difficulty of precisely identifying the initial conditions of practice makes the outcome hardly predictable. The study of movement variability can help to estimate a certain level of complexity of the motor system, and for this purpose a variety of tools from nonlinear dynamics and chaos theory (Slifkin \& Newell, 1998) are used to examine the issue of the degrees of freedom in movement control (based on Bernstein, 1967).

\subsubsection{Entropy measure}

Rooted in the information theory (Schneider \& Sagan, 2005), a first approach to study the complex nature of a time series is to assess the amount of disorder in this series. For this purpose, Entropy indexes the predictability of a time series, with high predictability indicating low disorder and vice versa. The measurement of entropy indicates to what extent one can predict the next state of a system, taking into account one's knowledge of its current state (Kantz \& Schreiber, 2004). Based on the concept of entropy, Pincus (1991) developed a measure called approximate entropy (ApEn) in order to calculate the predictability of relatively short empirical time series. If the value of $\mathrm{ApEn}$ is close to zero, the time series is very regular, predictable, and less complex (i.e. the next observation is easily predictable from the previous observations). Conversely, if ApEn is closer to two, the time series is more unpredictable, more random (i.e. it is difficult or impossible to predict the next observation from the previous ones). Pincus (2006) suggested that increased entropy refers to increased complexity by greater coupling between subsystems and fast and efficient communication. This measure of complexity has been applied to behavioural time series to investigate the regularity of centre of pressure fluctuations (Cavanaugh, et al., 2005), cardiovascular dynamics (Tulppo, et al., 2001), and isometric force production (Slifkin \& Newell, 1999). Over the years, several methods of entropy calculation have emerged (e.g. fuzzy entropy, conditional entropy, compression entropy, multiscale entropy), but in all cases the information is held in the degree of complexity of the time series (see Bravi, Longtin, \& Seely, 2011 for a precise presentation of all methods).

\subsubsection{Recurrence quantification analysis}

A nonlinear method called Recurrence plots was developed to study time series (Eckmann, Kamphorst, \&
Ruelle, 1987) and has been successfully used in a variety of disciplines, from psychology to climate change. In simple terms, a recurrence plot is nothing more than the visualization of a recurrence matrix, plotting the same time series in the $x$ axis and the $y$ axis. If the value on the $x$ axis and the value on the $y$ axis are considered as identical or "neighbours", the point of intersection is defined as recurrent. However, matches between points do not always have to be exact, and matching sequences are identified within a tolerance defined by the experimenter. Yet a recurrence plot is in fact not quite so simple because it is computed on the basis of the reconstruction of the movement attractor into the phase space (regarding a set of embedded parameters, see Marwan, Carmenromano, Thiel, \& Kurths, 2007, for a tutorial). The phase space is reconstructed by taking the measured signal and a number of time-lagged copies of this signal, with the number of copies defined as the dimension of the created phase space. This notion of phase space is based on the idea that the measured signal is influenced by many dynamical variables, and since all the influencing variables of a system cannot be measured (or even known), the reconstructed phase space with time-lagged observables enables researchers to investigate the true system dynamics by preserving key invariants of the dynamics (see Riley, et al., 1999, for an example).

Recurrence plots therefore capitalize on the property of dynamical systems to repeat over time (i.e. to recur) (Kuznetsov, et al., 2014). They make it possible to visualize how the states of a dynamical system evolve over time and in this way can reveal hidden patterns in irregular and complex time series. Based on recurrence plots, Webber and Zbilut (1994) and Zbilut and Webber (1992) developed specific recurrence features that could be automatically and objectively extracted from the plots in order to quantify the nature of the variability in the process; in other words, to quantify possible hidden patterns. The main (but not exhaustive) extractions of this recurrence quantification analysis (RQA) are: the Recurrence Rate (i.e. how often the system visits the same state over time), Determinism (how often the same sequences of states repeat identically), Maximum and Mean Diagonal Length (how long these repeated sequences are), Entropy (how many different patterns of repeated segments there are), and Trend (whether the time series is stationary or derives).

In this example (Fig. 2), the time series that were not differentiated by standard deviation measurements (Fig. 1) are now clearly differentiable by the recurrence plot methods and thereafter by recurrence quantification indicators. RQA therefore provides a wide range of indicators informing about the nature of the variability within a time series, taking into account the order of the data points. It has been successfully but little used so far in the movement and sport sciences (e.g., Cotuk \& Yavuz, 2007; Riley, et al., 1999; Riley \& Clark, 2003), probably because it requires the identification of a substantial number of input parameters to be appropriately used. 

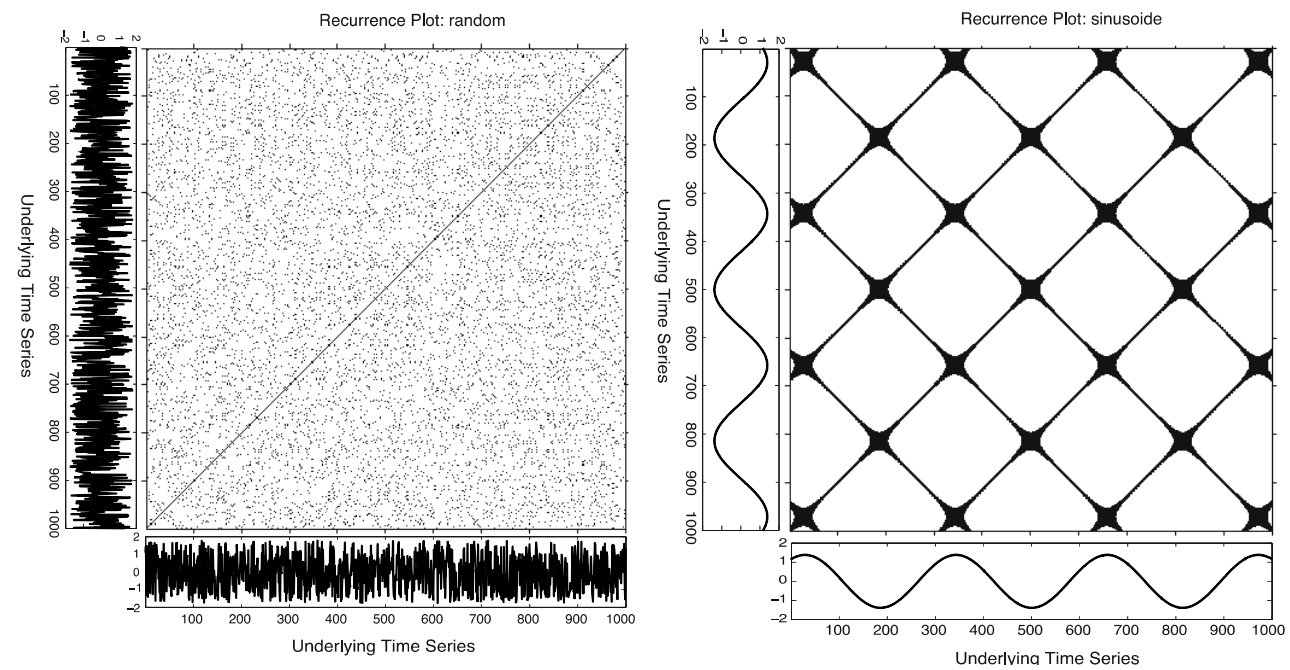

Fig. 2. Examples of recurrence plots based on a random signal (left) and a sinusoidal signal (right) (dimension $=2$, time lag $=$ $0.04 \mathrm{~s}$, radius $=10 \%$ of the mean size of the phase space). A black dot at the intersection of both underlying time series (in $x$ and $y$ ) refers to identical values (i.e. recurrent points); a white dot at the intersection refers to different values.

\subsubsection{Largest Lyapunov exponent}

Rather than predictability or the recurrence of data points within a time series, the largest Lyapunov exponent (LLe) provides a measure of the rate of divergence of infinitesimally close trajectories within a system. In other words, rather than assessing the amount or the nature of variability of a movement system (i.e. the variance from a reference), LLe assesses how fast nearby trajectories within the system deviate from a certain orbit (i.e. also called local stability), thereby providing information about system stability (i.e. the dynamic ability to offset a perturbation). As with recurrence quantification analysis, LLe calculation first requires the reconstruction of the attractor within the phase space, and trajectories here are defined within the phase space. Then, when LLe is computed, a positive value means that in the case of a slightly different position at instant $t$, this difference will increase with time (i.e. the trajectories within the phase space will diverge), and the higher the LLe, the faster the divergence will be. Usually, a positive LLe is associated with sensitivity to the initial conditions and is one of the features of the presence of chaos in the system (Cignetti, Decker, \& Stergiou, 2012). A value close or equal to zero reflects low or no divergence in the trajectories, indicating a highly stable movement system. In human movement studies, LLe has therefore been used as a direct measure of movement stability/instability (Dingwell \& Cusumano, 2000) (see Decker, Cignetti, \& Stergiou, 2012 for precision about the calculation).

\subsection{Discrete data analysis}

In the inter-trial or inter-subject context, the measurement methods aim at discriminating movement profiles rather than characterizing the nature of the variability within a time series. Profiles can be determined from the kinetics of kinematic variables (e.g. joint angular displacement, limb positions) of many individuals who have repeated the same movement under the same task conditions (e.g. Schöner \& Kelso, 1988; Seifert, et al., 2011) or within a single individual but over many trials (e.g. many repetitions performed during motor learning; Komar, Hérault, \& Seifert, 2013). As the variance did in quantifying the variability while omitting the position of the values, several tools derived from this statistical variance have been developed to characterize the amount of variability between signal waveforms, namely considering the position of the values in time (e.g. root mean square, Cauchy criterion). In addition, other procedures from machine learning (e.g. cluster analysis, neural networks) and robotic path planning (e.g. uncontrolled manifold) can help to define the nature of inter-trial variability.

\subsubsection{Normalized root mean square}

An extension of the standard deviation calculation was specifically applied to discrete movement variability analysis by Sidaway, Heise, and Schoenfelder-Zohdi (1995), who presented a technique called Normalized Root Mean Square (NoRMS) for measuring the consistency or variability of angle-angle plots (or time-angle plots). The NoRMS approach measures the averaged value of the variability between different signals and the mean pattern at each instant of the trial. Basically, the calculation is based on the squared Euclidean distance between two patterns of movement at each point that is averaged, and the square root is taken as the NoRMS (see Rein, 2012, for the equation). Hence, a lower NoRMS value for one individual compared with others indicates lower 
movementvariability for this individual. The main problem with this type of variability measurement is that the averaged movement is by definition virtual: the variability is calculated with regard to a pattern that has no real existence. Nevertheless, NoRMS has been used for a variety of movement patterns defined by time-continuous data (Chow, et al., 2007; Hodges, Hayes, Horn, \& Williams, $2005)$ in a wide variety of investigations: motor learning, clinical, and sport expertise, among others.

\subsubsection{Cauchy criterion}

Chen, Liu, Mayer-Kress, and Newell (2005) used a quasisimilar approach in a study on learning the pedalo locomotor task, but rather than making comparisons with an average pattern, the comparison was made with the previous cycle or trial. The Cauchy criterion is based on differences in movement patterns in space-time, and if the movement trajectories in two successive trials are similar, the value of the Cauchy criterion is low. Conversely, a high value means that two successive trials are quite different. This approach avoids the problem of making comparisons with a virtual average pattern, but still informs only about the quantity of variability (Rein, 2012). Indeed, in the case of consecutive high values of the Cauchy criterion, there is no information about the possibility of a drift in the movement pattern (i.e. successive trials going further and further from the initial one) as opposed to a mere fluctuation (i.e. subsequent trials going back and forth to the initial one). In any case, when it was applied to a pedalo locomotor task, this indicator informed about a decrease in the variability of movement trajectories during learning (Chen, et al., 2005).

Thus far, we have seen that measuring variability for discrete data is a way to assess the relative consistency of a movement pattern with regard to a predetermined reference or model (e.g. averaged pattern, criterion pathway, previous cycles). As highlighted by Rein (2012), these indicators can be characterized as discrete summary statistics as they encapsulate a complex phenomenon into a quantitative value of variability. The complex systems approach, however, is able to not only quantify variability but also investigate its nature.

\subsubsection{Cluster analysis}

Cluster analysis was initially developed to deal with problems of data mining and pattern recognition in high dimensional data sets, and it has since been used in a variety of experiments in movement science (e.g. Chow, Davids, Button, \& Koh 2008; Seifert, et al., 2011). An essential feature of this method is that no prior information is entered into the analysis. Generally, common algorithms like hierarchical agglomerative cluster analysis use information on similarities in order to group objects into clusters in an iterative stepwise manner. This type of algorithm will therefore group trials into ever larger clusters and the experimenter has to choose the appropriate number of clusters as part of the analysis result (usually represented through a dendrogram, Fig. 4). Another common method known as portioning or $k$-means consists of partitioning the input data sets into $n$ number of clusters, with $n$ defined beforehand by the experimenter. In all cases, the output of the analysis is a label assigned to each input trial that defines the trial as belonging to a specific cluster. The average pattern of each output cluster allows the researcher to qualify the movement variability between emerging clusters. Therefore, instead of looking at significant differences between pre-labelled experts and novices, as is done with hypothesis-testing statistics, cluster analysis merely groups similar input data without prior information about who is an expert and who is a novice. This unsupervised character of cluster analysis seems promising for movement variability experiments, especially if we consider that an individual's label as an expert or novice is very often based on a performance variable, whereas the dependent variables of the statistics concern movement organization (e.g. Komar, Sanders, Chollet, \& Seifert, 2014). Cluster analysis may therefore be an interesting exploratory process for potentially grouping the trials within a large data set, without knowledge about the number or even the existence of the groups.

To perform this analysis, each trial or individual can be defined by several variables, the number of which is defined by the experimenter, but it must be kept in mind that including unrelated variables might have a negative impact on the quality of the analysis. An advantage is that the input variables can stem from many domains, and may include interval, ordinal, nominal or ratio-scaled scales (sometimes requiring prior normalization) (see Rein, Button, Davids, \& Summers, 2010 for an in-depth methodological review). Interestingly for movement science, time-continuous data like kinetic movement patterns can also be clustered. For instance, Lee, Chow, Komar, Tan, and Button (2014) examined the movement variability of the forehand in 24 tennis players based on 12 time-continuous kinematic variables for each player. Moreover, advanced cluster algorithms allow feature selection. In other words, some cluster algorithms (e.g. Fisher-EM, Bouveyron \& Brunet, 2011) provide output with both a label for each trial and information about the weight of each input variable in discriminating the emerging clustering (i.e. how discriminant each dependent variable is in defining the clusters). For instance, Komar, Hérault, Seifert (2013) defined both the coordination patterns that appeared during breaststroke learning and the key features of the breaststroke cycles that were highly discriminant during learning.

A major issue in cluster analysis is that the algorithms always group objects into clusters, even when the data have no common structure. Indeed, the main point to keep in mind when using cluster analysis on experimental data is that the true grouping is usually unknown, thereby making it difficult to always have confidence 
in the results of the analysis. To overcome this limitation, validation procedures and indexes, usually derived from model-selection procedures (e.g. Bayesian Information Criterion, Akaike Information Criterion, CalinskyHarabasz; Calinski \& Harabasz, 1974), have emerged to help experimenters to strengthen the output of the clustering procedure. The general approach for selecting an appropriate number of clusters is based on the Fisher information; namely, the number of clusters that best fits the input data sets is the one that maximizes the intercluster distance while minimizing the intra-cluster distance. This approach seems particularly interesting for movement profiling, investigating the variability between trials and/or individuals, and assessing how these trials or individuals differ. Nevertheless, it does not directly inform about how good or functional the observed variability is.

\subsubsection{Uncontrolled manifold}

The uncontrolled manifold (UCM) technique was developed by Scholz and Schöner (1999) and is based on the notions of good variability and bad variability. In a task where the individual has to stabilize a particular value of a performance variable, the main assumption of UCM is that variability is good when the overall performance remains constant, whereas variability is bad when the performance deviates from the desired value. A good example from Latash, Scholz, and Schöner (2002) concerns a force production task, for which the individual is asked to press with two fingers to produce a $10 \mathrm{~N}$ force, 100 times. In every trial, the sum of the force produced individually by each finger has to be $10 \mathrm{~N}$, and as long as the variability in the force produced by each finger is such that the sum remains constant, the variability is good (e.g. finger one produces $4 \mathrm{~N}$ when finger two produces $6 \mathrm{~N}$ ). Conversely, when the variability in the force of each finger results in a sum divergent from $10 \mathrm{~N}$, the variability is considered as bad (e.g. finger one produces $5 \mathrm{~N}$ when finger two produces $6 \mathrm{~N})$. This phenomenon can be qualified as a type of synergy (Latash, et al., 2002), with one finger continuously compensating and adapting to the other, and vice versa, in order to ensure task success. The UCM approach is of paramount importance to the concept of functional variability, as it quantifies the good or functional part of variability, which is not directly possible with other methods (Rein, 2012). Here it becomes clear that the traditional interpretation of movement variability as error has lost its predominance because the UCM framework supports the view that variability has an inherent structure that can ensure functional adaptation in order to ensure task success (Davids, et al., 2003). In practice, this means that there is not merely one solution (e.g. $5 \mathrm{~N}$ produced by each arm), but a real set of solutions. For instance if one arm shows a maximal force production around $3 \mathrm{~N}$ because of fatigue, the other arm can compensate by producing higher force to keep the sum of both arms at $10 \mathrm{~N}$. Wu and Latash (2014) reported interesting results about mo- tor learning, showing that an increase in good variability led to improved performance.

Undeniably, a wide range of tools can be used to quantify and qualify movement variability or stability. Yet in some specific cases like investigating variability of a fixedpoint attractor in a dynamical system perspective, the information extracted from the amount of variability directly relates to the stability of the system. But this is not always the case and for instance when the system is assumed as chaotic-like, variability and stability/instability are not directly comparable as they represent different properties of the motor system (see Stergiou \& Decker, 2011 , for a discussion). In other words, the measure of variability seems highly dependent of the specific system that is actually investigated. Although we used the umbrella term 'variability' to introduce our topic, readers should be attentive to the properties actually measured through each tool (e.g., regularity, stability, amount of variability), because even though they are in some way all linked to the issue of motor variability, the information they provide might not always be exactly the same. In the second part of this paper, we highlight how these tools have helped to reconsider the role of movement variability as fundamental in defining motor expertise.

\section{Perspectives in interpreting motor variability}

In this section, we show how a complex systems perspective on motor variability and the associated tools have helped researchers to reconsider the traditional definition of expertise in the sport and movement sciences. From the outset, variability in movement organization and its potential adaptive and functional role needed to be carefully distinguished from variability in performance output, which is synonymous with inconsistency and therefore less functional (Davids, Bennett, \& Newell, 2006). This distinction has important implications for interpreting the quality of movement patterns that may deviate from a putative expert model of performance. Indeed, any consideration of the functional role of movement variability inevitably leads to an exploration of what adaptive behaviour means, and at the behavioural and functional scale it may be more appropriate to use the term adaptability rather than variability. Adaptability refers to a subtle ratio between stability (i.e. persistent behaviour despite external disturbance) and flexibility (i.e. variable behaviour responding to a dynamic environment) (Davids, et al., 2003). Even though expert movement patterns show regularities and similarities within their structural components, they are not rigidly fixed into a single solution but can be adapted in a functional way, since complex neurobiological systems can exploit inherent degeneracy (Edelman \& Gally, 2001). Neurobiological degeneracy is defined as 'the ability of elements that are structurally different to perform the same function or 

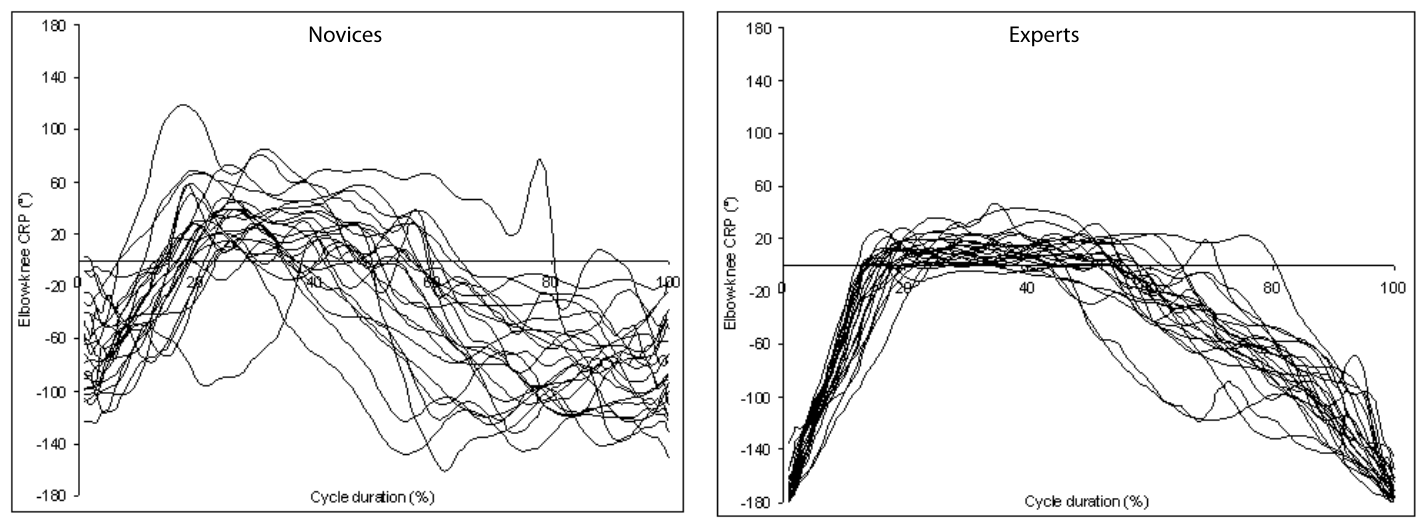

Fig. 3. Continuous relative phase $(\mathrm{CRP})$ between knee and elbow oscillations $\left(0^{\circ}=\right.$ in-phase coordination, $180^{\circ}$ and $-180^{\circ}=$ anti-phase coordination) for novice (left) and expert (right) swimmers. Each curve represents the time-normalized coordination during one cycle of one swimmer (i.e. from one leg maximal flexion to the next maximal leg flexion) (reprint from Seifert, et al., 2011).

yield the same output' (Edelman \& Gally, 2001, p. 13763). In complex biological systems, degeneracy provides the neurophysiological basis for the diversity of actions required to deal with an information-rich, dynamic environment from moment to moment (Seifert, et al., 2013). Degeneracy thus refers to the ability of an individual to vary motor behaviour structurally without compromising function, this being evidence of the adaptive and functional role of movement variability. Specifically, in this section we emphasize, i) how inter-individual variability may be a sign of adaptability to interacting constraints in swimming, ii) how an innovative pedagogical approach promoting variability during learning can cater for interindividual differences in tennis, and iii) the interest of investigating postural control and the potential functional role of centre of pressure variability.

\subsection{Inter-individual variability and interacting constraints in breaststroke swimming}

Although the classical hypothesis-testing approach seems to be the benchmark, cluster analysis is now often used to examine movement pattern variability for a range of movement skills such as walking, kicking, throwing or basketball shooting (Rein, et al., 2010; Chow, Davids, Button, \& Rein, 2008; Schorer, Baker, Fath, \& Jaitner, 2007). Seifert, et al. (2011) used this statistical approach to reconsider motor expertise in breaststroke swimming with regard to movement patterning rather than performance outcome. In this experiment, arm-leg coordination was investigated for 24 recreational and 24 expert swimmers, each with their level of expertise defined beforehand by their personal best performance. All the swimmers were then asked to perform a $25-\mathrm{m}$ swim at $80 \%$ of their maximal individual speed (therefore, an identical task regardless of individual skill levels). During the swim, elbow and knee angles were assessed for each individual using an underwater video camera, and the arm-leg coordination was calculated based on the continuous relative phase (CRP) between elbow and knee oscillations. The analysis therefore consisted in comparing the CRP curves of 24 recreational swimmers and the CRP curves of 24 expert swimmers (Fig. 3).

The results showed significant differences in the key points of the CRP curves between the expert and recreational swimmers. Interestingly, the authors thereafter focused on the high variability exhibited by the recreational swimmers (Fig. 3, left panel). Indeed, the variability exhibited within the recreational swimmer group seemed at some points greater than the variability between the recreational and expert swimmers. The authors then performed a cluster analysis on the same data and, whereas the first statistical approach indicated a difference between the recreational and expert swimmers, the cluster analysis revealed four types of motor organization. The recreational group comprised three distinct coordination profiles, with the dendrogram showing how the 24 novices were grouped based on the distance between the individuals' coordination variables (Fig. 4).

In this study, Seifert, et al. (2011) used cluster analysis to investigate inter-individual variability and therefore gained insight into the nature of the different observed patterns. Rather than focusing on the novices' deviations from the expert pattern, the authors then discussed each profile and suggested that these swimmers were at different stages of acquiring expertise and/or perceived the task constraints or the water resistance differently. For instance, the swimmers of cluster three exhibited inphase coordination, which reflects a superposition of contradictory actions (i.e. arm propulsion with leg recovery). This coordination mode entails a freezing of degrees of freedom, as observed on a ski-simulator (Vereijken, van Emmerik, Whiting, \& Newell, 1992). Conversely, the swimmers of cluster 1 exhibited only partial superposition of contradictory actions and a higher peak of angular velocity during knee extension (see Seifert, et al., 2011, 


\section{Dendrogram}

Ward linkage; Euclidean distance

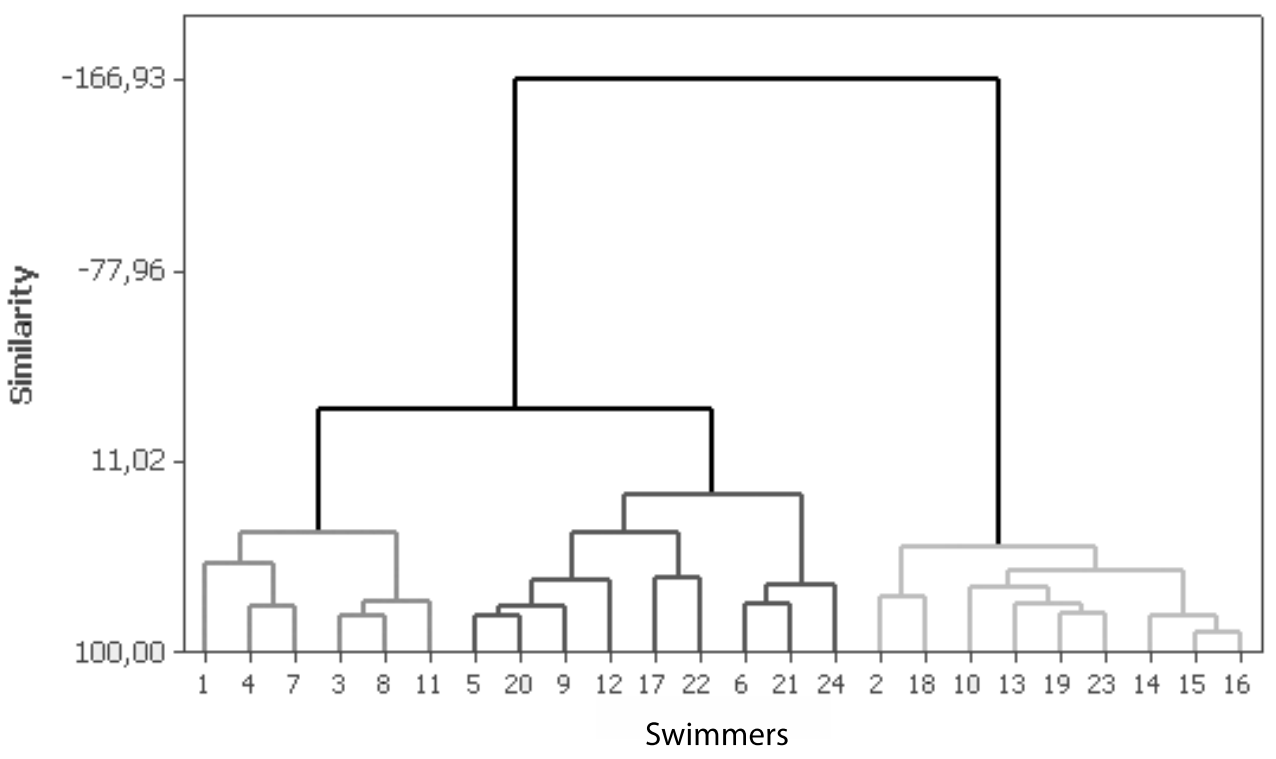

Fig. 4. Output dendrogram from Hierarchical Ascendant Clustering performed on the coordination of the 24 novice swimmers (Cluster $1=$ swimmers $[1,4,7,3,8,11]$, Cluster $2=$ swimmers $[5,20,9,12,17,22,6,21,24]$, Cluster $3=$ swimmers $[2,18,10$, 13, 19, 23, 14, 15, 16]). From the bottom to the top, each swimmer, initially alone, is grouped with his/her nearest neighbour until they are all grouped into one cluster. The height of each horizontal bar represents the distance between the two elements it joins (reprint with authorization, from Seifert, et al., 2011).

for greater detail). Specifically, because the swimmers in the three clusters were performing at the same level, the variability reflected inherent neurobiological degeneracy (Seifert, et al., 2013). This variability was thus considered as functional because it allowed the authors to examine the individual differences (e.g. anatomical interindividual differences); it also supported the functional exploration of the perceptual-motor workspace (e.g., exploring the Archimedes principle in swimming), a key feature of the complex systems account of motor learning (Newell, McDonald, \& Kugler, 1991). A key notion for this type of approach is that motor expertise might be less a question of acquiring the 'correct' movement pattern and more a question of finding the best adapted pattern to deal with the set of constraints acting on the performer at a given point in time (Davids, Button, \& Bennett, 2008).

\subsection{Promoting inter-individual differences in tennis learners}

A recent study used an advanced algorithm for cluster analysis (Bouveyron \& Brunet, 2011) to focus on the concept of exploratory motor learning and a nonlinear pedagogical approach to promoting inter-individual variability (Lee, et al., 2014). Nonlinear pedagogy is an innovative approach that advocates the manipulation of key task constraints specifically so that the learner can explore functional movement solutions while learning (Chow, et al., 2007). In a study of learning the tennis forehand stroke (Lee, et al., 2014), cluster analysis identified degeneracy by identifying different movement patterns during pre-, post- and retention-tests. In other words, the authors were interested not only in the nature of the movement patterns, but also in the number of emerging patterns at each step of the acquisition process. In this sense, the experiment showed that the nonlinear pedagogical approach increased inter-individual variability from four clusters at pre-test to seven clusters at posttest. Conversely, a linear pedagogical approach decreased the number of emerging clusters from four at pre-test to three at post-test. The authors thus concluded that nonlinear pedagogy was an effective way to cater for individual differences in learning a sport skill, whereas a linear/traditional pedagogy tended to promote the acquisition of more stereotyped behaviour.

An interesting aspect of this study was the high number of input variables the authors used for the cluster analysis. The dependent variables were the 12 timecontinuous joint angular positions recorded over the entire forehand stroke (e.g. right and left elbows, right and left shoulders, thorax and pelvis rotation), meaning that each learner was identified by 12 time series at each measurement timepoint. From these 12 dependent variables, the algorithm revealed how discriminative each joint was in the analysis. For example, the left and right shoulder rotation angles and the racquet rotation angle 
differentiated the clusters most of all. In other words, these joint angles were identified as showing the greatest change during learning. A practical implication of this finding might be to have tennis instructors focus learners' attention on these characteristics in order to improve the learning rate and/or optimality.

\subsection{Functional organization of the postural control system}

Postural control has traditionally been characterized within a biomechanical framework using the Sensory Organization Test (SOT, see Guskiewicz, Perrin, \& Gansneder, 1996 for an example of the protocol). The output of this test is a score estimating the maximum anterior-posterior angular displacement of the centre of gravity based on the displacement of the centre of pressure (COP). Hence, a higher score means lower amplitude and is assumed to refer to greater postural stability. Within this framework, traditional linear measures have been used extensively to analyse postural control (e.g., mean and peak velocity and displacement; Negahban, et al., 2009; Pfusterschmied, et al., 2013). Recently, however, the issue was raised of using analytic techniques that yield more than data on the gross properties of postural sway, but instead shed light on the underlying control processes (Riley, et al., 1999). This interest in new techniques was prompted by the observation that the traditional approach to postural stability, with its measures of velocity and displacement, was unlikely to detect profound changes in the nature of postural control (Stergiou \& Decker, 2011). Nonlinear analysis has thus been used in the study of postural control by RQA analysis of the COP displacements. For instance, Riley and Clark (2003) studied postural sway during the SOT, extending previous results focused on the length of the COP trajectory path. Usually, postural sway is least (i.e. in terms of surface and displacement length) when the eyes are open and the surface and visual surround are stable. Conversely, postural sway is greater when information from one of these sensory systems is missing and greatest when two sources of sensory information are missing (Peterka \& Black, 1990). When visual or somatosensory information are unavailable or altered, Riley and Clark (2003) found that postural sway becomes more regular, in addition to the changes in the amount of variability of the $\mathrm{COP}$ (i.e. surface and displacement). In other words, RQA analysis highlighted an increase in the percentage of recurrence in the displacement of COP, as well as an increase in the percentage of determinism. As previously suggested (Riccio, 1993), the authors concluded that postural sway creates sensory stimulation that may be used for balance control. Here, the increase in the amount of variability coupled with the increase in regularization might enhance the flow of information when the availability of sensory information is altered. In this sense, both the amount and the structure of variability might play a functional role in the control of stance. Another suggestion was that in the case of an increase in body sway in degraded sensory conditions, a simplification of this sway (i.e. an increase in regularity) is a compensatory strategy to simplify the sway pattern. In this case, the changes in the amount of variability are no longer functional, but the changes in the structure of this variability might still be highly functional (Riley \& Clark, 2003). Here, the combination of RQA and traditional linear analysis to study the temporal structure of COP variability provided interesting insight into the functional organization of the postural control system.

\section{Conclusion}

In this review, we sought to highlight how complex systems theory promotes a new perspective on movement variability by bringing to the forefront some of its functional aspects. Conversely to Ericsson's view of expertise, with its focus on error reduction and the reproduction of a putative expert model (Ercisson, Krampe, \& TeschRömer, 1993), a complex systems perspective prompts movement scientists to explore the functional role of variability and thereby contributes to redefining what an "expert" is. Indeed, the investigation of inter-individual variability has revealed some of the highly individualized motor responses that experts display in response to their unique individual constraints (e.g. anatomical, cognitive). The study of intra-individual variability continues to be useful as a means to determine the quality of a given performer's motor adaptability. In other words, expertise may refer more (or at least as much) to the ability to adapt to environmental or task constraints (e.g. opponents in football, speed changes during locomotion, lack of incoming information in postural balance) than to the ability to reproduce a predetermined (or stereotyped) motor pattern. If expertise resides more in the ability to switch effectively between movement patterns than in the nature of the patterns in themselves, notions about how expertise is acquired may need to be reconsidered, with the focus shifted to enhancing intra-individual variability during the learning process in order to improve later movement adaptability. For instance, recent investigations on nonlinear pedagogy (e.g. Komar, Chow, Chollet, \& Seifert, 2014; Lee, et al., 2014) have shown that promoting intra-individual variability during practice is likely to favour inter-individual variability in movement patterns after many weeks of practice, therefore respecting the individual constraints acting on each performer.

For both researchers and practitioners, it might be well to reconsider the research methodology in movement science and to complete the traditional linear analysis of the amount of variability with analytic techniques that provide access to the embedded structure of movement variability. Although they may at first glance seem too complex to implement, original methodologies (at least, original in movement science) like cluster analysis, recurrence quantification analysis and the uncontrolled 
manifold technique have demonstrated their power for movement analysis in both sport and medicine. In particular, these tools have helped to raise interesting questions and have yielded theoretical (e.g. functioning of postural control system) and practical (e.g. nonlinear pedagogy) directions for future explorations of the functional role and uses of movement variability in the definition and acquisition of movement expertise.

\section{Bibliography}

Bachelard, G. (1971). La formation de l'esprit scientifique (p. 304). Paris : Vrin.

Bernstein, N.A. (1967). The control and regulation of movements. London: Pergamon Press.

Biryukova, E.V, \& Bril, B. (2002). Bernstein et le geste technique. In V. Roux \& B. Bril (Eds.), Le geste technique. Réflexions Méthodologiques et Anthropologiques (pp. 4968). Erès.

Bouveyron, C., \& Brunet, C. (2011). Simultaneous modelbased clustering and visualization in the Fisher discriminative subspace. Statistics and Computing, 22 (1), 301-324.

Bravi, A., Longtin, A., \& Seely, A.J.E. (2011). Review and classification of variability analysis techniques with clinical applications. Biomedical Engineering Online, 10 (1), 90.

Calinski, T., \& Harabasz, J. (1974). A dendrite method for cluster analysis. Communications in Statistics - Theory and Methods, 3 (1), 1-27.

Cavanaugh, J.T., Guskiewicz, K.M., Giuliani, C., Marshall, S., Mercer, V., \& Stergiou, N. (2005). Detecting altered postural control after cerebral concussion in athletes with normal postural stability. British Journal of Sports Medicine, 39 (11), 805-811.

Chen, H.-H., Liu, Y.T., Mayer-Kress, G., \& Newell, K.M. (2005). Learning the pedalo locomotion task. Journal of Motor Behavior, 37 (3), 247-256.

Chow, J.Y., Davids, K., Button, C., \& Koh, M. (2007). Variation in coordination of a discrete multiarticular action as a function of skill level. Journal of Motor Behavior, 39 (6), 463-479.

Chow, J.Y., Davids, K., Button, C., \& Koh, M. (2008). Coordination changes in a discrete multi-articular action as a function of practice. Acta Psychologica, 127 (1), 163176.

Chow, J.Y., Davids, K., Button, C., \& Rein, R. (2008). Dynamics of movement patterning in learning a discrete multiarticular action. Motor Control, 12 (3), 219-240.

Chow, J.Y., Davids, K., Button, C., Shuttleworth, R., Renshaw, I., \& Araújo, D. (2007). The Role of Nonlinear Pedagogy in Physical Education. Review of Educational Research, 77 (3), 251-278.

Cignetti, F., Decker, L.M., \& Stergiou, N. (2012). Sensitivity of the Wolf's and Rosenstein's algorithms to evaluate local dynamic stability from small gait data sets. Annals of Biomedical Engineering, 40 (5), 1122-30.
Cotuk, B., \& Yavuz, E. (2007). Recurrence plot analysis of successive passing sequences in 2006 World Championship. Journal of Sports Science and Medicine, 6 (suppl. 10), 4.

Davids, K., Bennett, S.J., \& Newell, K.M. (2006). Movement System Variability. Champaign, Il.: Human Kinetics.

Davids, K., Button, C., \& Bennett, S.J. (2008). Dynamics of skill acquisition: A constraints-led approach. Champaign, IL: Human Kinetics.

Davids, K., Glazier, P.S., Araújo, D., \& Bartlett, R.M. (2003). Movement systems as dynamical systems: the functional role of variability and its implications for sports medicine. Sports Medicine, 33 (4), 245-260.

Davids, K., Hristovski, R., Araùjo, D., Balague Serre, N., Button, C., \& Passos, P. (2014). Complex systems in sport. New York, NY: Routledge.

De Rosnay, J. (1975). Le macroscope (p. 337). Paris: Seuil.

Decker, L.M., Cignetti, F., \& Stergiou, N. (2012). Wearing a safety harness during treadmill walking influences lower extremity kinematics mainly through changes in ankle regularity and local stability. Journal of Neuroengineering and Rehabilitation, 9, 8.

Dingwell, J.B., \& Cusumano, J.P. (2000). Nonlinear time series analysis of normal and pathological human walking. Chaos (Woodbury, N.Y.), 10 (4), 848-863.

Eckmann, J.P., Kamphorst, S.O., \& Ruelle, D. (1987). Recurrence plots of dynamical systems. Europhysics Letters, 4, 973-7.

Edelman, G.M., \& Gally, J.A. (2001). Degeneracy and complexity in biological systems. Proceedings of the National Academy of Sciences of the United States of America, 98 (24), 13763-8.

Ericsson, K.A., Krampe, R.T., \& Tesch-Römer, C. (1993). The role of deliberate practice in the acquisition of expert performance. Psychological Review, 100, 363-406.

Fitts, P.M. (1964). Perceptual-motor skills learning. In A.M. Melton (Ed.), Categories of Human Learning. New York, NY: Academic Press.

Fonseca, S., Diniz, A., \& Araújo, D. (2014). The Measurement of space and time in evolving sport phenomena. In $\mathrm{K}$. Davids, R. Hristovski, D. Araújo, N. Balague Serre, C. Button, \& P. Passos (Eds.), Complex systems in sport (p. 346). New York, NY: Routledge.

Gibson, J. (1979). The Ecological Approach to Visual Perception. Boston: Houghton Mifflin.

Guskiewicz, K.M., Perrin, D.H., \& Gansneder, B.M. (1996). Effect of mild head injury on postural stability in athletes. Journal of Athletic Training, 31 (4), 300-6.

Haken, H., Kelso, J.A.S., \& Bunz, H. (1985). A theoretical model of phase transitions in human hand movements. Biological Cybernetics, 51 (5), 347-356.

Hodges, N.J., Hayes, S.J., Horn, R.R., \& Williams, A.M. (2005). Changes in coordination, control and outcome 
as a result of extended practice on a novel motor skill. Ergonomics, 48 (11-14), 1672-85.

Kantz, H., \& Schreiber, T. (2004). Nonlinear time series analysis. Cambridge, MA: Cambridge University Press.

Kelso, J.A.S. (1984). Phase transitions and critical behavior in human bimanual coordination. The American Journal of Physiology, 246 (6 Pt 2), R1000-4.

Kelso, J.A.S. (1995). Dynamic Patterns: the self-organization of brain and behavior. Cambridge, MA: MIT.

Kelso, J.A.S., DelColle, J., \& Schöner, G. (1990). Actionperception as a pattern formation. In M. Jeannerod (Ed.), Attention and performance XIII (pp. 139-169). Hillsdale, NJ: Erlbaum.

Komar, J., Chow, J.Y., Chollet, D., \& Seifert, L. (2014). Effect of analogy instructions with an internal focus on learning a complex motor skill. Journal of Applied Sport Psychology, $26,17-32$.

Komar, J., Hérault, R., \& Seifert, L. (2013). Key point selection and clustering of swimmer coordination through Sparse Fisher-EM. In European conference on machine learning and principles and practice of knowledge discovery in databases. Prague, Tchek Republic.

Komar, J., Sanders, R.H., Chollet, D., \& Seifert, L. (2014). Do qualitative changes in arm-leg coordination lead to effectiveness of aquatic locomotion rather than efficiency. Journal of Applied Biomechanics, 30 (2), 189-97.

Kugler, P.N., Kelso, J.A.S., \& Turvey, M.T. (1980). On the concept of coordinative structures as dissipative structures: I. Theoretical lines of convergence. Advances in Psychology, 1, 3-47.

Kuznetsov, N., Bonnette, S., \& Riley, M.A. (2014). Nonlinear time series methods for analysing behavioural sequences. In K. Davids, R. Hristovski, D. Araújo, N. Balague Serre, C. Button, \& P. Passos (Eds.), Complex Systems in Sport (p. 346). New York, NY: Routledge.

Latash, M.L., Scholz, J.P., \& Schöner, G. (2002). Motor control strategies revealed in the structure of motor variability. Exercise and Sport Science Reviews, 30 (1), 26-31.

Lee, M.C.Y., Chow, J.Y., Komar, J., Tan, C.W.K., \& Button, C. (2014). Nonlinear pedagogy: an effective approach to cater for individual differences in learning a sports skill. PloS One, 9 (8), e104744.

Marwan, N., Carmenromano, M., Thiel, M., \& Kurths, J. (2007). Recurrence plots for the analysis of complex systems. Physics Reports, 438 (5-6), 237-329.

Mason, P.H. (2010). Degeneracy at Multiple Levels of Complexity. Biological Theory, 5 (3), 277-288.

Muller, H., \& Sternad, D. (2014). Decomposition of variability in the execution of goal-directed tasks: Three components of skill improvement. Journal of Experimental Psychology: Human Perception and Performance, 30 (1), 212-233.

Negahban, H., Hadian, M.R., Salavati, M., Mazaheri, M., Talebian, S., Jafari, A.H., \& Parnianpour, M. (2009). The effects of dual-tasking on postural control in people with unilateral anterior cruciate ligament injury. Gait $\&$ Posture, 30 (4), 477-481.

Newell, K.M. (1991). Motor skill acquisition. Annual Review of Psychology, 42, 213-37.

Newell, K.M., \& Corcos, D.M. (1993). Variability and motor control. Champain, IL: Human kinetics.

Newell, K.M., McDonald, P.V, \& Kugler, P.N. (1991). The perceptual-motor workspace and the acquisition of skill. In J. Requin \& G.E. Stelmach (Eds.), Tutorials in motor neuroscience (pp. 95-108). Netherlands: Kluwer Academic.

Newell, K.M., \& Slifkin, A.B. (1998). The nature of movement variability. In J.P. Piek (Ed.), Motor Behavior and Human Skill: A multidisciplinary approach. New York, NY: Human Kinetics.

Nourrit, D., Delignières, D., Caillou, N., Deschamps, T., \& Lauriot, B. (2003). On discontinuities in motor learning: a longitudinal study of complex skill acquisition on a skisimulator. Journal of Motor Behavior, 35 (2), 151-170.

Peterka, R.J., \& Black, F.O. (1990). Age-related changes in human posture control: sensory organization tests. Journal of Vestibular Research: Equilibrium \& Orientation, 1 (1), $73-85$.

Pfusterschmied, J., Buchecker, M., Keller, M., Wagner, H., Taube, W., \& Müller, E. (2013). Supervised slackline training improves postural stability. European Journal of Sport Science, 13 (1), 49-57.

Pincus, S.M. (1991). Approximate entropy as a measure of system complexity. Proceedings of the National Academy of Sciences of the United States of America, 88 (6), $2297-301$.

Pincus, S.M. (2006). Approximate entropy as a measure of irregularity for psychiatric serial metrics. Bipolar Disorders, 8 (5 Pt 1), 430-40.

Prigogine, I. (1994). Les lois du chaos (p. 126). Paris: Flammarion.

Rein, R. (2012). Measurement methods to analyse changes in coordination during motor learning from a non-linear perspective. The Open Sports Sciences Journal, 5 (1), 36-48.

Rein, R., Button, C., Davids, K., \& Summers, J. (2010). Cluster analysis of movement patterns in multiarticular actions: a tutorial. Motor Control, 14 (2), 211-39.

Riccio, G.E. (1993). Information in movement variability about the qualitative dynamics of posture and orientation. In K.M. Newell \& D.M. Corcos (Eds.), Variability and motor control (pp. 317-357). Champaign, IL: Kinetics, Human.

Riley, M.A., Balasubramaniam, R., \& Turvey, M.T. (1999). Recurrence quantification analysis of postural fluctuations. Gait \& Posture, 9 (1), 65-78.

Riley, M.A., \& Clark, S. (2003). Recurrence analysis of human postural sway during the sensory organization test. Neuroscience Letters, 342 (1-2), 45-8.

Riley, M.A., \& Turvey, M.T. (2002). Variability and determinism in motor behavior. Journal of Motor Behavior, 34 (2), 99-125. 
Schmidt, R.A. (1975). A schema theory of discrete motor skill learning. Psychological Review, 82, 225-260.

Schmidt, R.A. (2003). Motor schema theory after 27 years: reflections and implications for a new theory. Research Quarterly for Exercise and Sport, 74 (4), 366-75.

Schneider, E.D., \& Sagan, D. (2005). Into the cool: energy flow, thermodynamics, and life (p. 378). Chicago: University of Chicago Press.

Scholz, J.P., \& Schöner, G. (1999). The uncontrolled manifold concept: identifying control variables for a functional task. Experimental Brain Research, 126 (3), 289-306.

Schöner, G., \& Kelso, J.A.S. (1988). Dynamic pattern generation in behavioral and neurl system. Science, 239, 1513-20.

Schorer, J., Baker, J., Fath, F., \& Jaitner, T. (2007). Identification of interindividual and intraindividual movement patterns in handball players of varying expertise levels. Journal of Motor Behavior, 39 (5), 409-421.

Seifert, L., Button, C., \& Davids, K. (2013). Key properties of expert movement systems in sport: an ecological dynamics perspective. Sports Medicine, 43 (3), 167-178.

Seifert, L., Leblanc, H., Herault, R., Komar, J., Button, C., \& Chollet, D. (2011). Inter-individual variability in the upper - lower limb breaststroke coordination. Human Movement Science, 30 (3), 550-565.

Shannon, C.E., \& Weaver, W. (1949). The Mathematical theory of communication. (Vol. 47, p. 144). Chicago: University of Illinois Press.

Shaw, R. (2003). The Agent - Environment Interface: Simon's Indirect or Gibson's Direct Coupling? Ecological Psychology, 15 (1), 37-106.

Shea, C.H., \& Wulf, G. (2005). Schema theory: a critical appraisal and reevaluation. Journal of Motor Behavior, 37 (2), 85-101.

Sidaway, B., Heise, G., \& Schoenfelder-Zohdi, B. (1995). Quantifying the variability of angle-angle plots. Journal of Human Movement Studies, 29, 181-197.
Slifkin, A.B., \& Newell, K.M. (1998). Is variability in human performance a reflection of system noise? Current Directions in Psychological Science, 7 (6) 170-177.

Slifkin, A.B., \& Newell, K.M. (1999). Noise, information transmission, and force variability. Journal of Experimental Psychology. Human Perception and Performance, 25 (3), 837-51.

Stergiou, N., \& Decker, L.M. (2011). Human movement variability, nonlinear dynamics, and pathology: is there a connection? Human Movement Science, 30 (5), 869-888.

Tulppo, M.P., Mäkikallio, T.H., Seppänen, T., Shoemaker, K., Tutungi, E., Hughson, R.L., \& Huikuri, H.V. (2001). Effects of pharmacological adrenergic and vagal modulation on fractal heart rate dynamics. Clinical Physiology, 21 (5), 515-523.

Varela, F. (1989). Autonomie et connaissance. Essai sur le vivant [Autonomy and knowledge: Essay about living system]. Paris : Seuil.

Vereijken, B., van Emmerik, R.E.A., Whiting, H., \& Newell, K.M. (1992). Free(z)ing degrees of freedom in skill acquisition. Journal of Motor Behavior, 24 (1), 133-142.

Von Bertalanffy, L. (1969). General system theory (p. 283). New York, NY: George Braziller.

Webber, C.L., \& Zbilut, J.P. (1994). Dynamical assessment of physiological systems and states using recurrence plot strategies. Journal of Applied Physiology, 76 (2), 965-73.

Wolpert, D.M. (1997). Computational approaches to motor control. Trends in Cognitive Sciences, 1 (6), 209-216.

Wu, Y.-H., \& Latash, M.L. (2014). The effects of practice on coordination. Exercise and Sport Sciences Reviews, 42 (1), 37-42.

Zbilut, J.P., \& Webber, C.L. (1992). Embeddings and delays as derived from quantification of recurrence plots. Physics Letters A, 171 (3-4), 199-203.

Zwirn, H.P. (2006). Les systèmes complexes (p. 224). Paris : Odile jacob. 